\title{
MRI-Based Neuroanatomical Predictors of Dysphagia after Acute Ischemic Stroke: A Systematic Review and Meta-Analysis
}

\author{
Heather L. Flowers ${ }^{a} \quad$ Stacey A. Skoretz ${ }^{a} \quad$ David L. Streiner ${ }^{b, ~ e ~}$ Frank L. Silver $^{c}$ \\ Rosemary Martino ${ }^{a, d}$ \\ Departments of a Speech Language Pathology and ${ }^{b}$ Psychiatry, University of Toronto, ' University of Toronto and \\ Toronto Western Hospital, 'Health Care and Outcomes Research, Toronto Western Research Institute, Toronto, Ont., \\ and ${ }^{\text {DDepartment }}$ of Psychiatry and Behavioural Neurosciences, McMaster University, Hamilton, Ont., Canada
}

\section{Key Words}

Dysphagia - Stroke $\cdot$ Ischemia $\cdot$ Magnetic resonance imaging

\begin{abstract}
Background: Considering that the incidence of dysphagia is as high as $55 \%$ following acute stroke, we undertook a systematic review of the literature to identify lesion sites that predict its presence after acute ischemic stroke. Methods: We searched 14 databases, 17 journals, 3 conference proceedings and the grey literature using the Cochrane Stroke Group search strategy and terms for MRI and dysphagia. We evaluated study quality using the Cochrane Collaboration's risk of bias tool and extracted individual-level data. We calculated relative risks in order to model dysphagia according to neuroanatomical lesion sites. Results: Of 964 abstracts, 84 articles met the criteria for full review. Of these 84 articles, 17 met the quality criteria. These 17 articles dealt exclusively with dysphagia after infratentorial stroke and provided MRI correlates of dysphagia for 656 patients. The incidence of dysphagia according to stroke region was $0 \%$ in the cerebellum, $6 \%$ in the midbrain, $43 \%$ in the pons, $40 \%$ in the medial medulla and $57 \%$ in the lateral medulla. Within these regions, pontine (relative risk 3.7, 95\% confidence interval 1.57.7), medial medullary (relative risk $6.9,95 \%$ confidence inter-
\end{abstract}

val 3.4-10.9) and lateral medullary lesions (relative risk 9.6, 95\% confidence interval 5.9-12.8) predicted an increased risk of dysphagia. Conclusions: We sought to develop a neuroanatomical model of dysphagia throughout the whole brain. However, the literature that met our quality criteria addressed the MRI correlates of dysphagia exclusively within the infratentorium. Although not surprising, these findings are a first step toward establishing a neuroanatomical model of dysphagia after infratentorial ischemic stroke and provide insight into the assessment of individuals at risk for dysphagia.

Copyright $\odot 2011$ S. Karger AG, Basel

\section{Introduction}

Dysphagia is a frequently occurring consequence of stroke. A recent systematic review identified an incidence of 55\% in the acute stage [1]. Dysphagia is associated with comorbidities such as malnutrition, aspiration pneumonia and death. A recent study showed that pneumonia was associated with poor outcomes such as daily depen-

Institutions where the review was conducted: University of Toronto and University Health Network, Toronto, Ont., Canada.

\section{KARGER}

Fax +41613061234 E-Mail karger@karger.ch www.karger.com

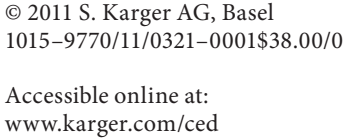

Heather L. Flowers, MEd, MHSc

Department of Speech Language Pathology, University of Toronto

160-500 University Avenue

Toronto, ON M5G 1 V7 (Canada)

Tel. +1 416946 3826, E-Mail heather.flowers@utoronto.ca 
dency or death after ischemic stroke [2]. Individuals with dysphagia have a risk of pneumonia 3 times higher than those without dysphagia [1]. Given these potential compromises, it is important to identify the precursors to dysphagia after stroke. Predicting the increased risk of dysphagia following brain lesions might increase our understanding of the causes of poststroke dysphagia, leading to improved management and better treatments for stroke survivors.

Recent advances in neuroimaging have made associating lesions with stroke severity possible [3-5]. Magnetic resonance imaging (MRI), including diffusion-weighted imaging (DWI) and T1- and T2-weighted scans, quantifies brain lesion sites and volumes, facilitating the diagnosis of stroke type, prior stroke and acute stroke. In particular, DWI is advantageous as it is most sensitive to hyperacute cerebral infarction $[6,7]$. A positive DWI scan distinguishes an acute infarct from an old infarct better than T2-weighted $[8,9]$ or computed tomography (CT) scans $[10,11]$. DWI scans are frequently acquired in the acute stage of stroke along with standard T1 and T2 scans [5]. Given the availability of these scanning techniques, researchers have begun to correlate MRI-based neuroanatomical findings with functional measures, such as the National Institutes of Health Stroke Scale [12].

Recent lesion-based studies have also associated dysphagia with distinct neuroanatomical regions following stroke. These areas include the frontal cortex [13], internal capsule [14, 15], insula [13, 16], periventricular white matter [17], thalamus [18], pons [19-23] and medulla [2434]. Taken together, these findings have the potential to form a synopsis of the neuroanatomical predictors of dysphagia. However, the reported incidence varied widely even with lesions in the same brain regions. In some cases, poor study quality contributed to such variation. For example, some studies documented dysphagia based on the report of symptoms or did not differentiate its incidence for hemorrhagic versus ischemic stroke. Similarly, instead of identifying the MRI-based correlates of dysphagia, many studies provided aggregate results from CT or MRI scans.

Given the discrepant incidence findings in the literature, we sought to systematically review and evaluate the evidence to derive a whole-brain model of dysphagia secondary to acute ischemic stroke. Our objectives were to identify published and unpublished literature correlating MRI-based lesions with dysphagia after acute ischemic stroke, to critically appraise the evidence and, if appropriate, to pool the available data to predict dysphagia subsequent to lesions in discrete brain regions.

\section{Materials and Methods}

We conducted this review systematically and according to published guidelines [35], starting with a detailed protocol developed a priori and proceeding to a comprehensive search of sources. Next, we assessed the study quality of accepted articles and finally extracted our targeted data from them.

\section{Operational Definitions}

We defined dysphagia to be 'oropharyngeal dysphagia', characterized by abnormal swallowing physiology of the upper aerodigestive tract and detected by clinical examination or instrumental testing [1]. The outcome of interest included MRI-based neuroanatomical lesion sites associated with the presence or absence of dysphagia. We restricted the sample to ischemic stroke, defined as an acute focal neurological deficit with a cerebral infarct confirmed by MRI imaging.

\section{Data Sources}

Database Searches

We searched 14 databases for abstracts published in any language between 1950 and June 2009, using the Cochrane Stroke Group Database Search Protocol [35]. The databases were AMED, Biosis, CENTRAL, CINAHL, Compendium, Dissertation Abstracts, Embase, HAPI, Healthstar, Inspec, Medline, Pharmaceutical Abstracts, PsychInfo and Wilson. We used the same medical subject headings and text word search terms as the Cochrane Stroke Group for each database with customizations. That is, we accepted all study designs and added search terms for dysphagia and MRI. The search included archived terms for MRI, such as 'magnetic resonance spectroscopy', 'nuclear magnetic resonance imaging', 'proton spin tomography' and 'zeugmatography'.

Journal, Conference and Grey Literature Searches

We manually searched 17 relevant journals, including Cerebrovascular Diseases, Dysphagia and Stroke, from 1991 to June 2009, and 3 conference proceedings (Dysphagia Research Society, the European Stroke Meeting and the International Stroke Conference) from the first date of their online availability to June 2009. We also searched the System for Information on Grey Literature to identify unpublished and grey literature.

\section{Eligibility Criteria}

Eligible articles used MRI to associate neuroanatomical lesion sites with the presence or absence of dysphagia. We included studies in any language with adult ischemic stroke patients ( $>18$ years). We accepted the following study designs: (1) case series with $\geq 10$ consecutively enrolled patients, (2) case-control studies, (3) prospective or retrospective cohort studies with consecutive enrolment, and (4) randomized controlled trials. If consecutive enrolment was unclear for the case series or cohort articles, we contacted the authors to confirm it. Consequently, we excluded (1) editorials and review articles, (2) studies with restricted samples (e.g. referrals to a speech-language pathology service), (3) studies without extractable dysphagia outcomes by brain region, and (4) studies without extractable MRI outcomes for ischemic stroke.

Data Retrieval

One author (H.L.F.) independently searched the data sources to retrieve all relevant citations. Two authors (H.L.F. and S.A.S.) 
then independently evaluated abstracts of the retrieved citations to select articles for full review. Bilingual clinical experts translated the French, German, Japanese and Spanish articles. Subsequently, two authors (H.L.F. and S.A.S.) independently evaluated the full articles to determine the final selection for quality review and data extraction. Discrepancies in abstract or article selection were resolved by consensus.

\section{Data Abstraction}

Study Quality

We used relevant items from the Cochrane Collaboration's risk of bias tool to evaluate study quality [36]. Specifically, we rated all selected articles for blinding and completeness of data for the dysphagia outcome. We documented additional factors including study design, consistency of the dysphagia assessment for all patients and use of operational definitions for dysphagia. Two authors (H.L.F. and S.A.S.) independently evaluated factors as present, absent or not reported (unclear). Again, discrepancies in the evaluation of quality factors were resolved by consensus.

\section{Study Characteristics and Outcomes}

In keeping with our protocol, one author (H.L.F.) extracted data on study characteristics, dysphagia and MRI outcomes. Another author (R.M.) independently checked the accuracy of the extracted data for $15 \%$ of the articles. The targeted study characteristics included the total number of enrolled patients, the length of the enrolment period, the targeted brain regions and the country in which the study was conducted. We categorized articles according to their capture of a first-ever ischemic stroke sample or a mixed ischemic stroke sample. We classified articles as dealing with first-ever stroke if the number of patients with recurrent stroke was $\leq 5 \%$ of the sample. Mixed stroke articles had $>5 \%$ recurrent stroke patients or the authors did not clearly describe the proportion of first-ever versus recurrent stroke. We also extracted information about the MRI scans and dysphagia assessments, including the timing of the MRI scan and dysphagia assessment following stroke onset, the MRI scan type used for lesion analyses and the method of the dysphagia examination. In addition, we extracted all available individual-level data from each selected article, including the presence or absence of dysphagia, age, gender and site and side of the neuroanatomical lesion.

\section{Statistical Analysis}

We pooled individual-level data to derive the frequency of dysphagia according to neuroanatomical region. We defined dysphagia frequency to be the first identification of dysphagia based on clinical records or prospective evaluation [1]. To test the presence versus absence of dysphagia between groups, we used two-tailed independent-sample $t$ tests for continuous variables. For frequency-based variables, we used $\chi^{2}$ analyses. Evaluation of these group differences guided our logistic regression modeling. We proposed to control for group differences, should they exist, with regard to age, gender and dysphagia assessment type (videofluoroscopy versus bedside evaluation). For measures of effect, we computed odds ratios and relative risks along with their $95 \%$ confidence intervals. Using clinical rationale, we selected predictor regions that we expected to have a high incidence of dysphagia. We performed analyses using SAS 9.2 to derive the odds ratios, subsequently con- verting them into relative risks for ease of interpretation [37]. We then developed a neuroanatomical model of dysphagia after acute ischemic stroke and tested our model for its prediction of dysphagia using Nagelkerke's $\mathrm{R}^{2}$ test.

\section{Results}

\section{Literature Retrieved}

There were a total of 1,110 citations relating to dysphagia, stroke and MRI from our database searches $(n=766)$, manual journal searches $(n=250)$ and conference proceedings $(\mathrm{n}=94)$. Of all the citations, 964 had full abstracts, of which 880 did not meet our study inclusion criteria (fig. 1). We selected the remaining 84 abstracts for full article review. There were 76 English articles, 4 Japanese, 2 Spanish, 1 French and 1 German. Following full review, we eliminated 67 articles (fig. 1). Two articles were randomized controlled trials [38, 39]. We eliminated them because MRI outcomes were not reported. We finally accepted 17 articles for this review, 15 in English and 2 in Japanese.

\section{Methodological Quality and Characteristics of Included Studies \\ Study Quality}

All but one article explicitly stated consecutive enrolment of patients. For the remaining article [33], we contacted the first author, who verified consecutive enrolment. All studies used a cohort design, but only 4 stated $[23,29,40,41]$ the timeline for data capture (table 1). Only 1 article reported blinding for the dysphagia measure [31]. Four studies described the same dysphagia assessment protocol for all patients; 1 involved screening [30], 1 videofluoroscopy [31] and 2 informal clinical evaluation $[26,42]$. Only 2 studies declared an operational definition for their dysphagia outcome [26, 30]. All but one of the selected articles had complete outcome data for dysphagia at the individual patient level. The article that failed to meet this criterion reported that 4 patients had dysphagia, but provided individual-level data documenting dysphagia for only 3 [40].

\section{Study Characteristics}

For the 17 articles, there was a total enrolment of 745 patients, with sample sizes ranging from 10 to 214 . The articles represented 6 countries, namely South Korea $(\mathrm{n}=8)$, Japan $(\mathrm{n}=3)$, Turkey $(\mathrm{n}=3)$, Spain $(\mathrm{n}=1)$, Switzerland $(n=1)$ and the USA $(n=1)$. Enrolment periods ranged from 1 year and 3 months to 8 years. The differ- 
Fig. 1. Selection process from abstract and

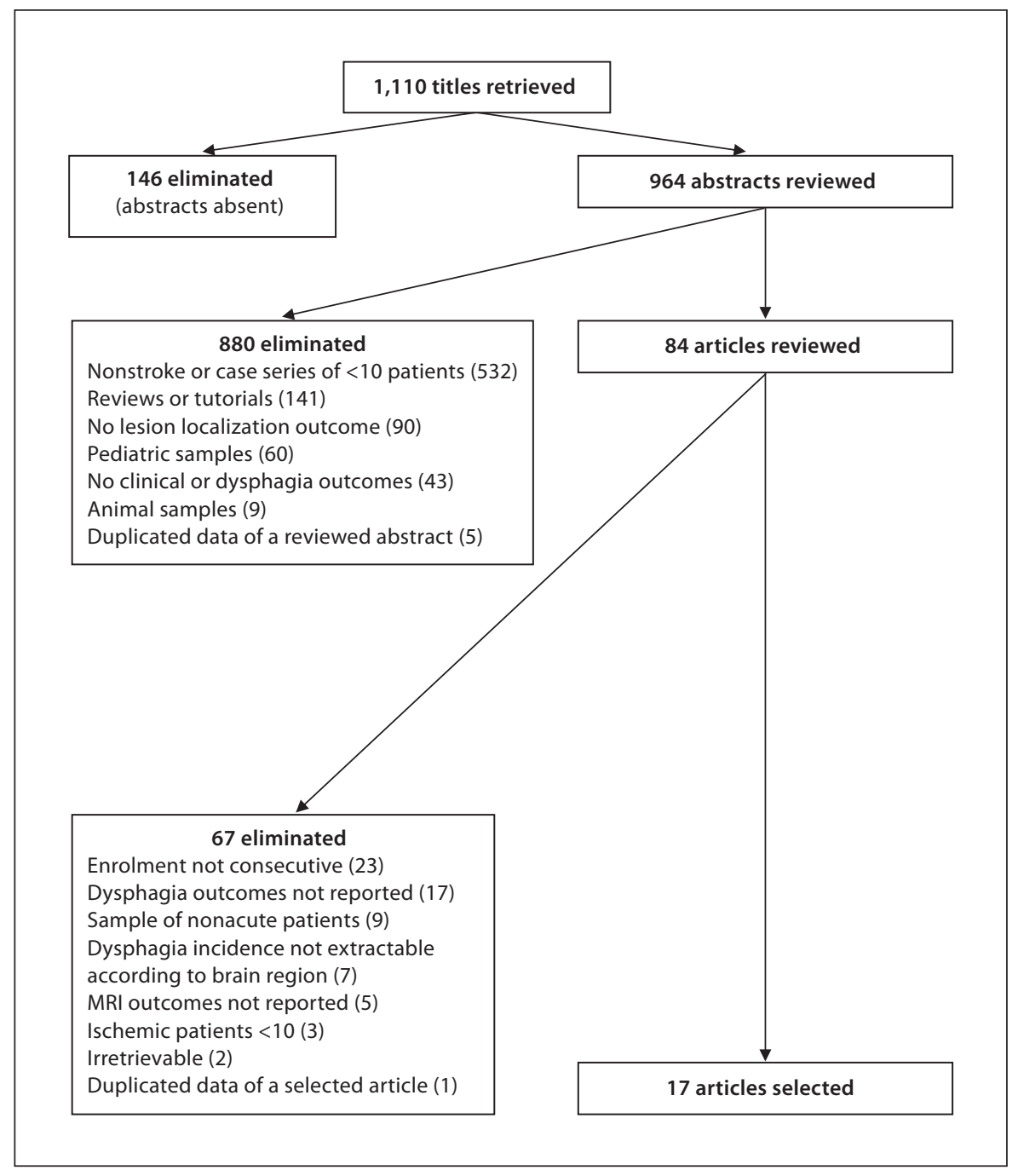
full article reviews.

ence in the frequency of dysphagia in patients with firstever stroke compared to those with mixed stroke was not significant $\left[\chi^{2}(4, \mathrm{n}=656)=0.72, \mathrm{p}=0.40\right]$.

\section{Neuroanatomical Regions of Interest}

All 745 patients from the 17 articles had sustained infratentorial strokes. The articles evaluated dysphagia after cerebellar, pontine, medial medullary and lateral medullary ischemic stroke, defining discrete anatomical regions of interest (table 2). Cerebellar regions included a rostral boundary from between the middle cerebellar peduncle and the anterolateral pons to a caudal boundary of the pontomedullary junction $[41,43]$. One article identified isolated lesions within the middle cerebellar peduncle [42]. The midbrain region involved a rostral boundary from just below the lower thalamus to a caudal boundary just above the midbrain-pontine junction [40, 44]. Pontine lesions included a rostral boundary of the round shape of the pons with a small round-shaped aqueduct [22] and a caudal boundary of just above the medulla [23], where images of the facial and acoustic nerves showed grooves [22]. Articles that reported lesions in the medulla identified regions extending from either the pontomedullary junction $[26,30,34]$ or the posterolateral bulging of the restiform body $[27-29,31,33,45]$ to the relatively round shape of the medulla with a closed fourth ventricle [26-28, 31, 33, 45] or to the cervicomedullary junction $[25,29,30,34]$. The lateral medullary regions did not include paramedian extension. Similarly, medial medullary regions did not include lateral extension.

Concerning their MRI scan protocols, 5 articles reported using T2, T1 and DWI scans [23, 31, 40, 43, 44]. 
Table 1. Evaluation of study quality regarding capture of dysphagia outcome

\begin{tabular}{|c|c|c|c|c|c|}
\hline Article & $\begin{array}{l}\text { Timeline for } \\
\text { data capture }\end{array}$ & $\begin{array}{l}\text { Assessor } \\
\text { blinded }\end{array}$ & $\begin{array}{l}\text { Consistent } \\
\text { assessment } \\
\text { for all patients }\end{array}$ & $\begin{array}{l}\text { Declared opera- } \\
\text { tional definition } \\
\text { for outcome }\end{array}$ & $\begin{array}{l}\text { Outcome } \\
\text { addressed for } \\
\text { all patients }\end{array}$ \\
\hline Izumi et al. [42] & unclear & unclear & yes & no & yes \\
\hline Kameda et al. [25] & unclear & unclear & unclear & no & yes \\
\hline Kim et al. [26] & unclear & unclear & yes & yes & yes \\
\hline Kim et al. [28] & unclear & unclear & unclear & no & yes \\
\hline Kim et al. [27] & unclear & unclear & unclear & no & yes \\
\hline Kim et al. [22] & unclear & unclear & unclear & no & yes \\
\hline Kim [45] & unclear & unclear & unclear & unclear & yes \\
\hline Kim and Kim [40] & mixed & unclear & unclear & no & no \\
\hline Kumral et al. [44] & unclear & unclear & unclear & no & yes \\
\hline Kumral et al. [29] & retrospective & unclear & unclear & no & yes \\
\hline Kumral et al. [43] & unclear & unclear & unclear & no & yes \\
\hline Kurono et al. [30] & unclear & unclear & yes & yes & yes \\
\hline Kwon et al. [31] & unclear & yes & yes & no & yes \\
\hline Min et al. [41] & prospective & unclear & unclear & no & yes \\
\hline Schmahmann et al. [23] & prospective & unclear & unclear & no & yes \\
\hline Valls-Solé et al. [33] & unclear & unclear & unclear & no & yes \\
\hline Vuilleumier et al. [34] & unclear & unclear & unclear & no & yes \\
\hline
\end{tabular}

Table 2. Study characteristics according to stroke type (first-ever or mixed) for all patients

\begin{tabular}{|c|c|c|c|c|c|c|c|c|}
\hline Article & $\begin{array}{l}\text { Number } \\
(\mathrm{n}=745)\end{array}$ & Country & $\begin{array}{l}\text { Enrolment } \\
\text { period, years; } \\
\text { months }\end{array}$ & $\begin{array}{l}\text { Targeted } \\
\text { brain } \\
\text { regions }\end{array}$ & $\begin{array}{l}\text { Days } \\
\text { to MRI }{ }^{1}\end{array}$ & $\begin{array}{l}\text { MRI lesion } \\
\text { analysis (thick- } \\
\text { ness, } \mathrm{mm} \text { ) }\end{array}$ & $\begin{array}{l}\text { Days to } \\
\text { dysphagia } \\
\text { exam }^{1}\end{array}$ & $\begin{array}{l}\text { Method of } \\
\text { dysphagia } \\
\text { exam }\end{array}$ \\
\hline \multicolumn{9}{|l|}{ First-ever stroke patients } \\
\hline Kim et al. [26] & 23 & South Korea & $3 ; 0$ & LM, MM & NR & NR & $\leq 14$ & VFS \\
\hline Kim et al. [27] & 18 & South Korea & $4 ; 2$ & MM & NR & $\mathrm{T} 2(5-6)$ & NR & clinical $^{2}$ \\
\hline Kim et al. [22] & 37 & South Korea & $3 ; 4$ & pons & NR & $\mathrm{T} 2(5-6)$ & NR & clinical \\
\hline Kim [45] & 130 & South Korea & $8 ; 0$ & LM & NR & $\mathrm{T} 2(3)$ & $\leq 7$ & clinical \\
\hline Kumral et al. [43] & 23 & Turkey & $2 ; 0$ & cerebellum & $\leq 3$ & DWI (5) & NR & clinical \\
\hline Kwon et al. [31] & 46 & South Korea & $1 ; 4$ & LM, MM & $\leq 11$ & $\mathrm{~T} 2(3)^{3}$ & $4.6 \pm 2.3^{4}$ & VFS \\
\hline Schmahmann et al. [23] & 25 & USA & $8 ; 0$ & pons & NR & NR & NR & clinical \\
\hline \multicolumn{9}{|l|}{ Mixed stroke patients } \\
\hline Izumi et al. [42] & 10 & Japan & $7 ; 0$ & cerebellum & $7-21$ & NR (NR) & $7-21$ & clinical \\
\hline Kameda et al. [25] & 214 & Japan & $5 ; 0$ & $\mathrm{LM}, \mathrm{MM}$ & NR & NR (NR) & NR & clinical \\
\hline Kim et al. [28] & 33 & South Korea & $3 ; 6$ & LM & NR & $\mathrm{T} 2(5-6)$ & $\leq 5^{5}$ & clinical \\
\hline Kim and Kim [40] & 40 & South Korea & $6 ; 9$ & midbrain & $\leq 10$ & NR (NR) & NR & clinical \\
\hline Kumral et al. [44] & 41 & Turkey & $6 ; 0$ & midbrain & $\leq 7$ & NR (NR) & NR & clinical \\
\hline Kumral et al. [29] & 11 & Turkey & $7 ; 0$ & $\mathrm{MM}$ & $\leq 7$ & NR (5) & NR & clinical \\
\hline Kurono et al. [30] & 21 & Japan & $3 ; 2$ & LM & NR & $\mathrm{T} 2$ (NR) & NR & screening \\
\hline Min et al. [41] & 31 & South Korea & $1 ; 3$ & cerebellum & NR & $\mathrm{T} 2(3)$ & NR & clinical \\
\hline Valls-Solé et al. [33] & 14 & Spain & NR & LM, MM & NR & $\mathrm{T} 2(5-6)$ & $\leq 7$ & clinical \\
\hline Vuilleumier et al. [34] & 28 & Switzerland & $3 ; 1$ & LM, MM & $\leq 16^{6}$ & NR (5) & $\leq 16$ & clinical \\
\hline \multicolumn{9}{|c|}{$\begin{array}{l}\text { The mixed stroke group includes articles in which }>5 \% \text { of the } \\
\text { patients had sustained a recurrent stroke. It also includes those } \\
\text { articles that did not clearly report a sample of first-ever stroke. } \\
\text { LM = Lateral medulla; } M M=\text { medial medulla; NR = not reported; } \\
\text { VFS = videofluoroscopy. }\end{array}$} \\
\hline
\end{tabular}


Seven articles used T1 and T2 scans [22, 27-29, 34, 41, 45] and 2 used T2 scans [30,33]. Three articles did not report their MRI scan protocol $[25,26,42]$. Eight articles used T2-weighted MRI scans for their lesion analyses [22, 26$28,30,31,33,41]$, while 1 used diffusion-weighted MRI scans [43]. The remaining 8 articles did not report the scan type used for lesion analyses $[23,25,26,29,34,40$, $42,44]$. Of all the articles, 7 described the timing of MRI scanning after stroke onset, ranging from within 3 [43] to 21 days [42].

\section{Dysphagia Outcomes Reported}

Seven of the 17 articles reported the timing of the dysphagia evaluation after the onset of stroke, ranging from within 5 [28] to 21 days [42] (table 2). No article reported the duration between MRI scanning and dysphagia assessment. Fourteen articles reported dysphagia evaluation through informal clinical examination $[22,23,25$, $27-29,33,34,40-45]$. Two reported dysphagia assessment by videofluoroscopy $[26,31]$, performed by a speech-language pathologist, with outcomes of laryngeal penetration or aspiration. One article used screening, thereby identifying the presence or absence of a risk of dysphagia [30].

\section{Incidence and Neuroanatomical Models of Dysphagia after Acute Ischemic Stroke}

All 17 selected articles had extractable individual patient data for their dysphagia measure with comparable reports of age and gender characteristics (table 3). We excluded patients who had received CT scans and those who had lesions extending from infratentorial regions into subcortical and/or cortical regions. From the initial sample of 745 patients, there were 656 eligible patients with circumscribed lesions in discrete regions. These regions included isolated lesions in the cerebellum, midbrain, pons or lateral or medial medulla and pontine, medial medullary or lateral medullary lesions extending only into the cerebellum. There were 469 males and $187 \mathrm{fe}$ males. In 248 patients, there were individual data for age, which ranged from 27 to 89 years (mean 61.8 years). The presence versus absence of dysphagia was not significant for gender $\left[\chi^{2}(4, \mathrm{n}=248)=1.47, \mathrm{p}=0.22\right]$ or age $[\mathrm{t}(\mathrm{d} . \mathrm{f}$. $=$ $246)=0.42, \mathrm{p}=0.68]$. Similarly, a $\chi^{2}$ analysis for dysphagia assessment type was not significant $\left[\chi^{2}(4, \mathrm{n}=515)=\right.$ $2.0, \mathrm{p}=0.16]^{1}$

\footnotetext{
1 Videofluoroscopy was used in 2 articles reporting medullary infarcts Consequently, we conducted the $\chi^{2}$ analysis in the sample of patients with medullary infarcts.
}

Individual-level data for the laterality of the lesion were available in 238 patients. Right-sided lesions occurred in 118 patients, left-sided in 112 and bilateral in 8 . Within this sample of 656 patients, the incidence of dysphagia according to stroke region was $0 \%$ in the cerebellum, $6 \%$ in the midbrain, $43 \%$ in the pons, $40 \%$ in the medial medulla and $57 \%$ in the lateral medulla (table 4). Our model demonstrated an increased risk of dysphagia after pontine, medial medullary and lateral medullary stroke compared to cerebellar or midbrain stroke (fig. 2). Nagelkerke's R ${ }^{2}$ was 0.203 .

Post hoc analyses for only the subgroup of patients with isolated lesions $(n=399)$ demonstrated an incidence of dysphagia according to stroke region of $0 \%$ in the cerebellum, $6 \%$ in the midbrain, $49 \%$ in the pons, $50 \%$ in the medial medulla and 59\% in the lateral medulla. The frequency of dysphagia in this subgroup compared to the whole sample was not statistically significant $\left[\chi^{2}(4, n=\right.$ $656)=3.38, p=0.067]$. As anticipated, a logistic regression model of the subgroup revealed comparable findings to those of the whole sample, with overlapping confidence intervals for prediction statistics.

\section{Discussion}

The goal of this review was to develop a whole-brain model of dysphagia. However, the available evidence was limited to infratentorial stroke, involving the cerebellum, midbrain, pons and medial and lateral medulla. Within these regions, the reported frequency of dysphagia ranged from 0 to $57 \%$ and was lowest after cerebellar lesions and highest after lateral medullary lesions. These findings suggest that cerebellar lesions did not contribute to the presence of dysphagia. In contrast, lesions in the pons and medial and lateral medulla were highly associated with the presence of dysphagia. Although these results are not surprising, they are the first step in quantifying the risk of dysphagia based on neuroanatomical lesion site.

This study is the first to predict the presence of dysphagia in a large sample of patients. We pooled results for this large sample of patients because there were individual-level data for dysphagia frequency in all articles. The samples were homogeneous with respect to their consecutive enrolment of patients and their selection of patients with acute ischemic stroke. In addition, all articles clearly reported which patients had received MRI scans. Our neuroanatomical model demonstrated a much higher likelihood of dysphagia after pontine and medullary le- 
Table 3. Characteristics according to brain region for eligible patients

\begin{tabular}{|c|c|c|c|c|c|c|}
\hline Brain region & $\begin{array}{l}\text { Eligible } \\
\text { patients } \\
(\mathrm{n}=656)\end{array}$ & $\begin{array}{l}\text { Males } \\
\mathrm{n}\end{array}$ & $\begin{array}{l}\text { Mean age } \\
\text { years } \\
\text { (range) }\end{array}$ & $\begin{array}{l}\text { Dysphagia after } \\
\text { isolated lesions } \\
\mathrm{n} / \mathrm{N}(\%)\end{array}$ & $\begin{array}{l}\text { Dysphagia after isolated } \\
\text { lesions extending into } \\
\text { the cerebellum, } \mathrm{n} / \mathrm{N}(\%)\end{array}$ & $\begin{array}{l}\text { Dysphagia fre- } \\
\text { quency for all eli- } \\
\text { gible patients, } \%\end{array}$ \\
\hline \multicolumn{7}{|l|}{ Cerebellum } \\
\hline Izumi et al. [42] & 7 & 3 & $56(46-76)$ & $0 / 7$ & - & 0 \\
\hline Kumral et al. [43] & 8 & 6 & NR (31-81) & $0 / 8$ & - & 0 \\
\hline Min et al. [41] & 21 & 15 & $59(42-78)$ & $0 / 21$ & - & 0 \\
\hline Total & & & & $0 / 36$ & - & 0 \\
\hline \multicolumn{7}{|l|}{ Midbrain } \\
\hline Kumral et al. [44] & 9 & 7 & $63(41-83)$ & $0 / 9$ & - & 0 \\
\hline Kim and Kim [40] & 40 & 23 & $65(47-81)$ & $3 / 40$ & - & 8 \\
\hline Total & & & & $3 / 49(6)$ & - & 6 \\
\hline \multicolumn{7}{|l|}{ Pons } \\
\hline Schmahmann et al. [23] & 24 & 14 & $61(32-82)$ & $10 / 15$ & $1 / 9$ & 46 \\
\hline Kim et al. [22] & 32 & 23 & $63(36-85)$ & $13 / 32$ & - & 41 \\
\hline Total & & & & 23/47 (49) & $1 / 9(11)$ & 43 \\
\hline \multicolumn{7}{|l|}{ Medial medulla } \\
\hline Vuilleumier et al. [34] & 1 & 1 & 51 & $1 / 1$ & - & 100 \\
\hline Kim et al. [26] & 4 & 2 & $58(27-89)$ & $2 / 4$ & - & 50 \\
\hline Valls-Solé et al. [33] & 5 & 3 & $73(59-89)$ & $5 / 5$ & - & 100 \\
\hline Kumral et al. [29] & 7 & 6 & $58(26-80)$ & $3 / 7$ & - & 43 \\
\hline Kwon et al. [31] & 9 & 9 & $58(38-69)$ & $7 / 9$ & - & 78 \\
\hline Kim et al. [27] & 13 & 10 & $64(41-75)$ & $1 / 12$ & $1 / 1$ & 15 \\
\hline Kameda et al. [25] & 41 & 32 & $65 \pm 12$ & - & $12 / 41^{1}$ & 29 \\
\hline Total & & & & $19 / 38(50)$ & $13 / 42(31)$ & 40 \\
\hline \multicolumn{7}{|l|}{ Lateral medulla } \\
\hline Valls-Solé et al. [33] & 9 & 5 & $65(38-82)$ & $5 / 6$ & $2 / 3$ & 78 \\
\hline Kim et al. [26] & 19 & 15 & $63(43-82)$ & $10 / 19$ & - & 53 \\
\hline Vuilleumier et al. [34] & 19 & 17 & $60(30-85)$ & $8 / 11$ & $1 / 8$ & 47 \\
\hline Kurono et al. [30] & 21 & 17 & $62(43-83)$ & - & $10 / 21^{2}$ & 48 \\
\hline Kim et al. [28] & 33 & 22 & $56(32-71)$ & $15 / 26$ & $5 / 7$ & 61 \\
\hline Kwon et al. [31] & 37 & 27 & $54(29-76)$ & $13 / 37$ & - & 35 \\
\hline Kim [45] & 130 & 90 & $57(28-84)$ & $84 / 130$ & - & 65 \\
\hline Kameda et al. [25] & 167 & 122 & $61 \pm 12$ & - & $95 / 167^{1}$ & 57 \\
\hline Total & & & & $135 / 229(59)$ & $113 / 206(55)$ & 57 \\
\hline
\end{tabular}

${ }^{1}$ Cerebellar extension was reported for at least some patients (exact numbers not provided).

${ }^{2}$ Cerebellar extension was unclear for all patients. NR $=$ Not reported.

Table 4. Frequencies, odds ratios and relative risks of dysphagia according to brain region after acute ischemic stroke
MRI-Based Predictors of Dysphagia after Stroke

\begin{tabular}{lcccll}
\hline Brain region & $\begin{array}{l}\text { Dysphagia } \\
\text { incidence, } \%\end{array}$ & Odds ratio & $95 \%$ CI & $\begin{array}{l}\text { Relative } \\
\text { risk }\end{array}$ & $95 \%$ CI \\
\hline Cerebellum & 0 & - & - & - & - \\
Midbrain & 6 & - & - & - & - \\
Pons & 43 & 4.5 & $1.5-13.7$ & 3.7 & $1.5-7.7$ \\
Medial medulla & 40 & 11.1 & $4.0-30.3$ & 6.9 & $3.4-10.9$ \\
Lateral medulla & 57 & 21.9 & $8.7-55.2$ & 9.6 & $5.9-12.8$ \\
\hline
\end{tabular}

$\mathrm{CI}=$ Confidence interval. 


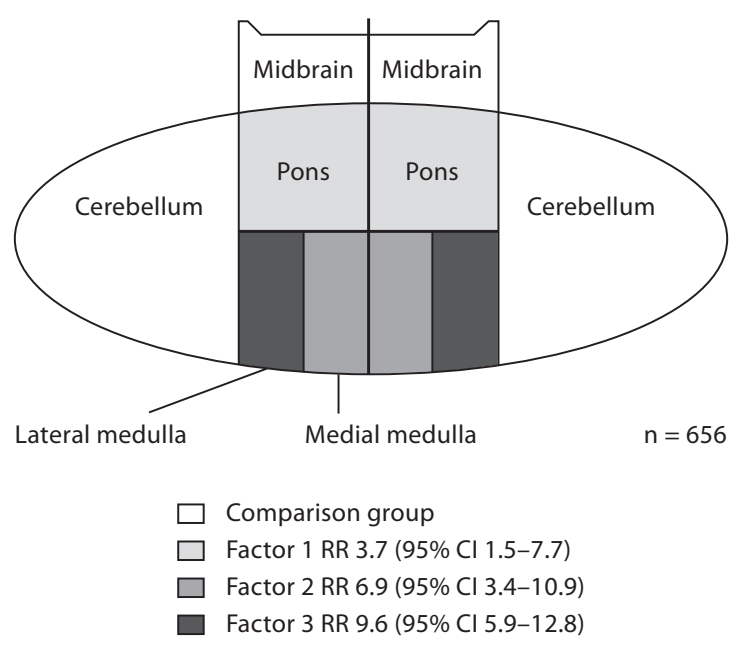

Fig. 2. Neuroanatomical model for dysphagia after acute ischemic infratentorial stroke (adapted from Broadley et al. [47]). RR = Relative risk; $\mathrm{CI}=$ confidence interval.

sions than after cerebellar or midbrain lesions. However, there were great discrepancies in the incidence of dysphagia after medial and lateral medullary lesions, ranging from $15 \%$ [27] to $100 \%[33,34]$ and from $35 \%$ [31] to $78 \%$ [33], respectively. These discrepancies may be due, in part, to differences in assessment methods and sample sizes. Although all studies evaluated acute stroke patients, there were inconsistencies in the timing and methodology of both the MRI imaging and the dysphagia assessments. Small sample sizes may limit the capture of true frequency; however, because we pooled individuallevel data, our model compensated for discrepancies in sample size.

The articles selected for this review were all observational and had methodological limitations. For example, all but one [31] lacked blinding for the dysphagia measure and only two $[26,30]$ used operational definitions for the presence or absence of dysphagia. The quality of the dysphagia assessments was poor, as no article reported reliability or validation of their measures. One article used a nonstandardized screening protocol [30], while two used a gold standard evaluation, namely videofluoroscopy [26, 31]. However, videofluoroscopic measures did not capture the entire swallow and were, instead, limited to laryngeal penetration or aspiration [26, 31]. The remaining articles reported informal clinical assessment, without clear documentation of their assessment procedures, limiting the reproducibility of their results. The articles we reviewed did not document the exact timing of the dysphagia evaluation on a case-by-case basis, thereby limiting the sensitivity of dysphagia outcomes. Most articles did not evaluate the lesion correlates of dysphagia using DWI scans for the hyperacute and acute phases of stroke. Despite these methodological shortcomings for the dysphagia outcome and MRI analysis, all articles provided clear documentation of the neuroanatomical regions of interest for their lesion analyses. In addition, most articles reported the range in timing of the dysphagia assessment and of the MRI scans for their samples.

A systematic review such as this is limited by the available evidence. The eligible articles reported only on dysphagia following infratentorial stroke. Articles dealing with supratentorial stroke were eliminated because they provided aggregate data for CT and MRI or did not differentiate between the dysphagia incidence following hemorrhagic versus ischemic stroke. Although not the purpose of the current systematic review, future work is needed to compare these findings with parallel bodies of literature identifying dysphagia after supratentorial stroke based on CT scans and after hemorrhagic stroke.

Not all of the articles we selected for our study reported age, gender, side of lesion or time to dysphagia assessment on an individual patient basis, limiting comparisons of the influence of these variables on the presence of dysphagia. Nevertheless, we identified detailed MRI information for a large number of patients, allowing us to establish a first robust model of dysphagia frequency following lesions within the infratentorium. This information is beneficial for the early detection of dysphagia after ischemic stroke, especially in light of current guidelines that recommend screening [46]. That is, knowing the relative risk of dysphagia according to lesion localization may precipitate earlier screening in patients with the highest risk. Consequently, this may facilitate more timely assessments by speech-language pathologists and treatments pertaining to implementing an oral diet or alternative mode of feeding.

\section{Conclusion}

The available literature did not address supratentorial lesions. Our results showed a high incidence of dysphagia following lesions located throughout the lower brainstem, especially in the lateral medulla. In contrast, dys- 
phagia was rare after a midbrain or cerebellar stroke. In the articles in this review, dysphagia was not assessed in a consistent manner or with objective methods. Some studies used bedside screening while others used informal clinical assessment, thereby limiting their ability to reliably and accurately detect the presence of dysphagia. Furthermore, studies that used the more objective method of videofluoroscopic assessment restricted their measures to laryngeal penetration or aspiration. Considering these gaps in the available literature, we conclude that fu- ture studies are needed to systematically and comprehensively evaluate dysphagia secondary to lesions throughout the whole brain.

\section{Acknowledgments}

We gratefully acknowledge Mitsuko Takeuchi's review and translation of the Japanese articles. We thank the many authors who responded to requests for additional information pertaining to their articles.

\section{References}

1 Martino R, Foley N, Bhogal S, Diamant N, Speechley M, Teasell R: Dysphagia after stroke: incidence, diagnosis, and pulmonary complications. Stroke 2005;36:2756-2763.

$\checkmark 2$ Vermeij FH, Scholte W, Reimer WJ, de Man P, van Oostenbrugge R, Frank C, de Jong G, de Kort P, Dippel D: Stroke-associated infection is an independent risk factor for poor outcome after acute ischemic stroke: data from the Netherlands Stroke Survey. Cerebrovasc Dis 2009;27:465-471.

3 Keir SL, Wardlaw JM: Systematic review of diffusion and perfusion imaging in acute ischemic stroke. Stroke 2000;31:2723-2731.

-4 Schaefer PW, Grant PE, Gonzalez RG: Diffusion-weighted MR imaging of the brain. Radiology 2000;217:331-345.

5 Tan PL, King D, Durkin CJ, Meagher TM, Briley D: Diffusion weighted magnetic resonance imaging for acute stroke: practical and popular. Postgrad Med J 2006;82:289-292.

- 6 Mullins M, Schaefer PW, Sorensen AG, Halpern EF, Ay H, He J, Koroshetz WJ, Gonzalez RG: CT and conventional and diffusion-weighted MR imaging in acute stroke: study in 691 patients at presentation to the emergency department. Radiology 2002; 224:353-360.

7 Prichard JW, Grossman RI: New reasons for early use of MRI in stroke. Neurology 1999; 52:1733-1736.

-8 Fazekas F, Niederkorn K, Ebner F, Diez-Tejedor E: Relevance of neuroimaging in the evaluation of cerebral ischemia. Cerebrovasc Dis 2009;27:1-8.

-9 van Everdingen KJ, van der Grond J, Kappelle LJ, Ramos LM, Mali WP: Diffusionweighted magnetic resonance imaging in acute stroke. Stroke 1998;29:1783-1790.

$\checkmark 10$ Barber PA, Darby DG, Desmond PM, Gerraty RP, Yang Q, Li T, Jolley D, Donnan GA, Tress BM, Davis SM: Identification of major ischemic change. Diffusion-weighted imaging versus computed tomography. Stroke 1999;30:2059-2065.
11 Lansberg MG, Albers GW, Beaulieu C, Marks MP: Comparison of diffusion-weighted MRI and CT in acute stroke. Neurology 2000;54:1557-1561.

12 Menezes NM, Ay H, Wang Zhu M, Lopez CJ, Singhal AB, Karonen JO, Aronen HJ, Liu Y, Nuutinen J, Koroshetz WJ, Sorensen AG: The real estate factor: quantifying the impact of infarct location on stroke severity. Stroke 2007;38:194-197.

13 Broadley S, Croser D, Cottrell J, Creevy M, Teo E, Yiu D, Pathi R, Taylor J, Thompson PD: Predictors of prolonged dysphagia following acute stroke. J Clin Neurosci 2003;10: 300-305.

14 Gonzalez-Fernandez M, Kleinman JT, Ky PK, Palmer JB, Hillis AE: Supratentorial regions of acute ischemia associated with clinically important swallowing disorders: a pilot study. Stroke 2008;39:3022-3028.

15 Hamdy S, Aziz Q, Rothwell JC, Power M, Singh KD, Nicholson DA, Tallis RC, Thompson DG: Recovery of swallowing after dysphagic stroke relates to functional reorganization in the intact motor cortex. Gastroenterology 1998;115:1104-1112.

16 Daniels SK, Foundas AL: The role of the insular cortex in dysphagia. Dysphagia 1997; 12:146-156.

17 Daniels SK, Foundas AL: Lesion localization in acute stroke patients with risk of aspiration. J Neuroimaging 1999;9:91-98.

18 Alberts MJ, Horner J, Gray L, Brazer SR: Aspiration after stroke: lesion analysis by brain MRI. Dysphagia 1992;7:170-173.

19 Bassetti C, Bogousslavsky J, Barth A, Regli F: Isolated infarcts of the pons. Neurology 1996;46:165-175.

20 Horner J, Buoyer FG, Alberts MJ, Helms MJ: Dysphagia following brain-stem stroke. Clinical correlates and outcome. Arch Neurol 1991;48:1170-1173.

21 Kim JS: Recurrent pontine base infarction: a controlled study. Cerebrovasc Dis 2002;13: 257-261.
22 Kim JS, Lee JH, Im JH, Lee MC: Syndromes of pontine base infarction: a clinical-radiological correlation study. Stroke 1995;26: 950-955.

23 Schmahmann JD, Ko R, MacMore J: The human basis pontis: motor syndromes and topographic organization. Brain 2004;127: 1269-1291.

-24 Aydogdu I, Ertekin C, Tarlaci S, Turman B, Kiylioglu N, Secil Y: Dysphagia in lateral medullary infarction (Wallenberg's syndrome): an acute disconnection syndrome in premotor neurons related to swallowing activity. Stroke 2001;32:2081-2087.

$>_{25}$ Kameda W, Kawanami T, Kurita K, Daimon M, Kayama T, Hosoya T, Kato T; Study Group of the Association of Cerebrovascular Disease in Tohoku: Lateral and medial medullary infarction: a comparative analysis of 214 patients. Stroke 2004;35:694-699.

26 Kim H, Chung CS, Lee KH, Robbins J: Aspiration subsequent to a pure medullary infarction: lesion sites, clinical variables, and outcome. Arch Neurol 2000;57:478-483.

27 Kim JS, Kim HG, Chung CS: Medial medullary syndrome: report of 18 new patients and a review of the literature. Stroke 1995;26: 1548-1552.

28 Kim JS, Lee JH, Suh DC, Lee MC: Spectrum of lateral medullary syndrome: correlation between clinical findings and magnetic resonance imaging in 33 subjects. Stroke 1994; 25:1405-1410.

29 Kumral E, Afsar N, Kirbas D, Balkir K, Ozdemirkiran T: Spectrum of medial medullary infarction: clinical and magnetic resonance imaging findings. J Neurol 2002;249: 85-93.

30 Kurono H, Uesaka Y, Kunimoto M, Imafuku I: The correlation between dysphagia and involvement of the ambiguous nucleus on MRI in acute-phase lateral medullary syndrome (in Japanese). Rinsho Shinkeigaku 2006;46: 461-466.

-31 Kwon M, Lee JH, Kim JS: Dysphagia in unilateral medullary infarction: lateral vs medial lesions. Neurology 2005;65:714-718. 
32 Seiser A, Brix U, Bosak P, Aichinger-Steiner MM, Brainin M: Wallenberg's lateral medullary infarction. Clinical features, neuroradiological findings and long term prognosis in 38 patients. Neuropsychiatrie 1999;13:917.

\$3 Valls-Solé J, Vila N, Obach V, Alvarez R, Gonzalez LE, Chamorro A: Brain stem reflexes in patients with Wallenberg's syndrome: correlation with clinical and magnetic resonance imaging (MRI) findings. Muscle Nerve 1996;19:1093-1099.

>34 Vuilleumier P, Bogousslavsky J, Regli F: Infarction of the lower brainstem. Clinical, aetiological and MRI-topographical correlations. Brain 1995;118:1013-1025.

35 Cochrane Stroke Group. Cochrane Review Groups. About The Cochrane Collaboration. http://onlinelibrary.wiley.com/o/cochrane/clabout/articles/STROKE/frame. html.
36 Higgins JPT, Green S (eds): Cochrane Handbook for Systematic Reviews of Interventions, version 5.0.2 (updated September 2009). The Cochrane Collaboration, 2009. Available from www.cochrane-handbook. org.

37 Zhang J, Yu KF: What's the relative risk? A method of correcting the odds ratio in cohort studies of common outcomes. JAMA 1998;280:1690-1691.

38 Dyker AG, Lees KR: Remacemide hydrochloride: a double-blind, placebo-controlled, safety and tolerability study in patients with acute ischemic stroke. Stroke 1999;30:17961801.

39 Kobayashi H, Nakagawa T, Sekizawa K, Arai H, Sasaki H: Levodopa and swallowing reflex. Lancet 1996;348:1320-1321.

40 Kim JS, Kim J: Pure midbrain infarction: clinical, radiologic, and pathophysiologic findings. Neurology 2005;64:1227-1232.

-41 Min WK, Kim YS, Kim JY, Park SP, Suh CK: Atherothrombotic cerebellar infarction: vascular lesion-MRI correlation of 31 cases. Stroke 1999;30:2376-2381.

42 Izumi M, Terao S, Sobue G, Koshimura J, Takatsu S, Yokoi Y, Takahashi M, Mitsuma $\mathrm{T}$ : Clinical features of anterior inferior cerebellar artery territory infarcts - a study of ten patients (in Japanese). No To Shinkei 1996; 49:152-156
43 Kumral E, Kisabay A, Atac C: Lesion patterns and etiology of ischemia in the anterior inferior cerebellar artery territory involvement: a clinical - diffusion weighted - MRI study. Eur J Neurol 2006;13:395-401.

-44 Kumral E, Bayulkem G, Akyol A, Yunten N, Sirin H, Sagduyu A: Mesencephalic and associated posterior circulation infarcts. Stroke 2002;33:2224-2231.

45 Kim JS: Pure lateral medullary infarction: clinical-radiological correlation of 130 acute, consecutive patients. Brain 2003;126:18641872 .

46 Lindsay PBP, Bayley MM, Hellings CB, Hill M, Woodbury E, Phillips S: Canadian best practice recommendations for stroke care (updated 2008). CMAJ 2008;179:E1-E93.

47 Broadley S, Cheek A, Salonikis S, Whitham E, Chong V, Cardone D, Alexander B, Taylor J, Thompson P: Predicting prolonged dysphagia in acute stroke: the Royal Adelaide Prognostic Index for Dysphagic Stroke (RAPIDS). Dysphagia 2005;20:303-310. 